\title{
A new approach to urinary bladder control with optogenetics
}

Optogenetics employs light to modulate cellular activities with high spatial and temporal precision. It has emerged as a powerful, novel technique for uncovering how neurons communicate with one another and is giving rise to more complex functions. The function of optogenetics is based on implementing certain light-sensitive transmembrane proteins, classified as opsins, to respond by changing their structural conformations under light with specific wavelengths ranging from 390 to $700 \mathrm{~nm}$, consequently promoting changes in ionic current flow across the cell membrane. For example, channelrhodopsin-2 (ChR2) is a light-gated ion channel that produces neuron depolarization with cation flow, which induces an excitatory response to illuminated, 470-nm wavelength blue light [1]. In contrast, halorhodopsin $(\mathrm{NpHR})$ is a light-sensitive chloride pump that provides an inhibitory response by hyperpolarization of the neurons transduced with 580-nm wavelength yellow light [2]. Overall, it is possible to transduce neurons with different types of opsins to stimulate or inhibit neuronal activity by exposing them to light pulses of certain specific wavelengths. Thus, those using this technique are empowered to avoid the nonspecific, broad effects of electrical stimulation or pharmacologic manipulation. Because the expression of the opsins is genetically targetable, the technique provides a high level of resolution by limiting only the neurons of interest to respond to the light.

Optogenetics was first reported in 2005 when it was shown that mammalian neuron action potentials can rapidly and steadily be controlled with millisecond precision [3]. Since then, various techniques and combined methods for optogenetics have emerged. In 2010, the journal Nature declared optogenetics to be the "method of the year." Now, optogenetics has found applications in various fields and has already changed how neuroscience research is conducted. Optogenetics has also made a significant contribution to our understanding of the mechanisms of and has laid down some groundwork for developing novel treatment protocols for several neurological disorders. There is a promising, optimistic view paired with application of the technology to improve the outcomes of conventional treatments in clinics in the near future. Initially, optogenetics was applied only to control neural circuits in the brain. Then, it was used to modulate the activities of the spinal cord and peripheral nervous system. In particular, optically controlling the peripheral nervous system associated with the urinary bladder has gained much attention in the last two to three years.

The urinary bladder is a major organ that is responsible for storing and voiding urine. Across several decades, there have been many attempts to control bladder function with various medical and surgical interventions, but most failed to yield clinically satisfying results. Recently, Park et al. [4] demonstrated that the smooth muscle cells (SMCs) of the bladder can be modulated using optogenetic membrane proteins. They applied the Creloxp transgenic system to deliver optogenetic membrane proteins, such as ChR2 and $\mathrm{NpHR}$, to mouse bladder SMCs. Then, using blue light, they induced the SMCs to depolarize via the ChR2 and employed yellow light to hyperpolarize them with $\mathrm{NpHR}$. Control of the bladder's contractile behaviors was thus a success using this method. Of note, these results could be the foundation for developing a novel treatment protocol for low urinary tract dysfunction such as overactive bladder and detrusor underactivity.

Optogenetics is also actively used in research aimed at modulating bladder pain. For example, Samineni et al. [5] explored the possibility of optogenetically inhibiting nociceptive sensory afferents to control bladder pain. In their research, they applied light-driven outward proton pumps known as archaerhodopsins (Arch) to inhibit certain sensory neurons. They ultimately discovered that optogenetically inhibiting nociceptive sensory afferents not only weakens 
the visceromotor response during bladder distention but also can reduce cystitis-associated consistent pain and cutaneous hypersensitivity as well. Their findings suggest that the optogenetic application of selective inhibition on nociceptive bladder afferents could be useful in attenuating and treating bladder pain.

Despite these successful results, however, optogenetic bladder modulation still faces some challenges. The bladder is a complex, dynamic, and heterogeneous system with muscles and nerves that make the delivery of light in freely moving animals to this area difficult. For instance, the muscle and connective tissue surrounding the bladder can block applied light, ultimately complicating optogenetic implementation. Therefore, in optogenetics research, it is important to find a way to achieve robust and consistent light irradiation to the bladder muscles or nerves in freely moving animals. Luckily, recent technological advancements in implantable optoelectronic systems can help to deliver optical stimulation to activate light-sensitive opsins in both the central and peripheral nervous systems. In a study of nociceptive sensory afferents, Samineni et al. [5] developed a fully implantable and wirelessly powered optoelectronic system that can consistently modulate the opsins expressed in bladder afferents. They also found that the optical simulation of nociceptive bladder afferents can reduce consistent bladder pain by applying the newly developed device in awake and moving animals.

Neuromodulation using electrical or optical stimulation is a technique that is used to alter the information flow within neural circuits for therapeutic benefits. This technique can generally be categorized either as an "open-loop" or "closedloop" paradigm. The existing open-loop neuromodulation approach has been successfully used as a treatment option for various neurological diseases, whereas the novel closedloop neuromodulation approach boasts many potentials for improving current patient treatments. Importantly, though, the latter also still faces challenges in improving the specificity of modulation and providing dynamic titration of stimulation with respect to the individual patient's needs.

In a recent study, a team of neuroscientists and engineers developed an approach combining various techniques from optogenetics, soft electronics, data analytics, wireless powering, and wireless communications in order to address overactive bladder syndrome in animals [6]. This "closed-loop" approach used real-time information to identify pathological voiding behavior and deliver a corrective signal in response. In detail, the researchers implanted a soft, stretchy belt-like device that wraps around the bladder. To act as a strain gauge, the implanted device increases its electrical resistance as the bladder becomes filled and decreases as the bladder is emptied. The researchers also injected a green lightsensitive opsin, Arch, to be expressed in the sensory neurons innervating the bladder neurons using a viral gene therapy vector. In their rat model study, the new device was designed to yield real-time information on bladder function and data algorithms for identifying pathological behavior. For instance, when the bladder was emptied too often, the device sent a signal that activated light-emitting diodes (LEDs) to focus on sensory neurons in the bladder. This procedure reduced sensory neuron activity and restored the bladder to normal function. When Arch were activated, such affected the neuronal membrane, causing the inhibition of action potentials. The LED activation further blocked specific neurons from sending signals from the bladder to the brain. This development could be used for people suffering from bladder problems and could even extend beyond the bladder to application in other tissues and organs of the body. Therefore, closed-loop optogenetic neuromodulation might be poised to play a key role in driving a transformative shift towards the use of such strategies to treat various disorders.

Although optogenetic modulation for urinary bladder conditions offers effectiveness and potentials, this method still faces several barriers to be overcome prior to actual widespread implementation in human patients. First, while there are clinical results regarding the short-term safety of transfecting human patients, the process of consistently and continuously expressing the transfected proteins is still in the early research stage. Considering the optoelectronic system, the application of optogenetic modulation in the human urinary bladder could demand more battery life/ power to support a larger light-generating implantable device, considering a human's bladder size and volume versus a small mammal's. Additionally, investigation into the body's long-term response to the presence of an implantable device that is wrapped around the bladder is necessary.

Recently, there have also been promising attempts made to introduce optogenetics as a novel treatment method for intractable diseases [7]. In August 2015, RetroSense Therapeutics (Ann Arbor, MI, USA), received United States Food and Drug Administration approval to move forward with human trials for treating blindness due to retinitis pigmentosa using a virus modified with ChR2 (ClinicalTrials. gov: NCT02556736). Separately, in 2018, GenSight Biologics (Paris, France) also received regulatory authorization in the United Kingdom for a phase I/II clinical trial for their eyewear device coupled with optogenetics (ClinicalTrials. gov: NCT03326336). The eyewear device is designed to restore vision in people who are blind as a result of retinitis 
pigmentosa. Such attempts demonstrate that the field of optogenetics is moving closer to clinical application. In the near future, optogenetic therapy for the management of urinary bladder diseases could also be applied to overcome the limits of existing treatment methods in the clinic.

\section{CONFLICTS OF INTEREST}

The authors have nothing to disclose.

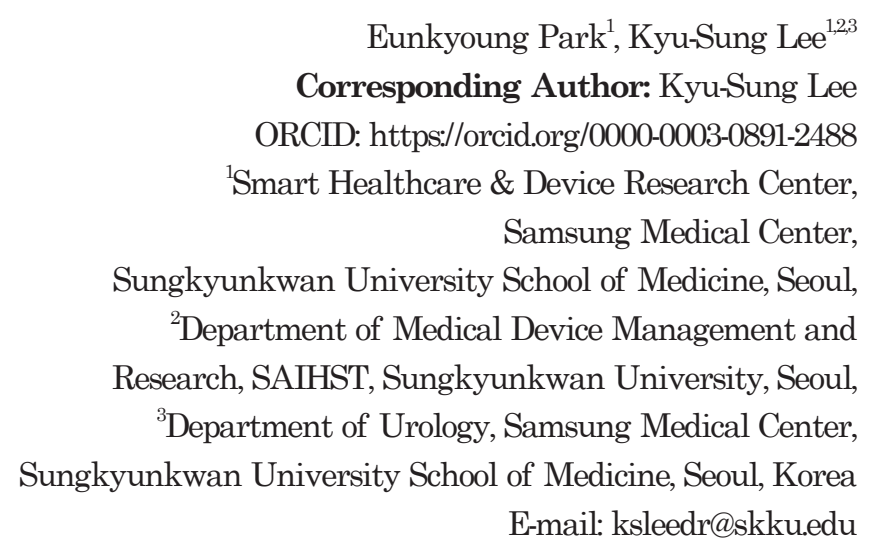

\section{REFERENCES}

1. Nagel G, Szellas T, Huhn W, Kateriya S, Adeishvili N, Ber- thold $\mathrm{P}$, et al. Channelrhodopsin-2, a directly light-gated cation-selective membrane channel. Proc Natl Acad Sci U S A 2003;100:13940-5.

2. Zhang F, Wang LP, Brauner M, Liewald JF, Kay K, Watzke N, et al. Multimodal fast optical interrogation of neural circuitry. Nature 2007;446:633-9.

3. Boyden ES, Zhang F, Bamberg E, Nagel G, Deisseroth K. Millisecond-timescale, genetically targeted optical control of neural activity. Nat Neurosci 2005;8:1263-8.

4. Park JH, Hong JK, Jang JY, An J, Lee KS, Kang TM, et al. Optogenetic modulation of urinary bladder contraction for lower urinary tract dysfunction. Sci Rep 2017;7:40872.

5. Samineni VK, Mickle AD, Yoon J, Grajales-Reyes JG, Pullen MY, Crawford KE, et al. Optogenetic silencing of nociceptive primary afferents reduces evoked and ongoing bladder pain. Sci Rep 2017;7:15865.

6. Mickle AD, Won SM, Noh KN, Yoon J, Meacham KW, Xue Y, et al. A wireless closed-loop system for optogenetic peripheral neuromodulation. Nature 2019;565:361-5.

7. Simunovic MP, Shen W, Lin JY, Protti DA, Lisowski L, Gillies MC. Optogenetic approaches to vision restoration. Exp Eye Res 2019;178:15-26. 